\title{
Reconstructing Cardiac Wave Dynamics From Myocardial Motion Data
}

\author{
Christopher B Beam ${ }^{1}$, Cristian A Linte ${ }^{2,3}$, Niels F Otani ${ }^{1}$ \\ ${ }^{1}$ School of Mathematical Sciences, Rochester Institute of Technology, Rochester, NY, United States \\ ${ }^{2}$ Biomedical Engineering, Rochester Institute of Technology, Rochester, NY, United States \\ ${ }^{3}$ Center for Imaging Science, Rochester Institute of Technology, Rochester, NY, United States
}

\begin{abstract}
Various models exist to predict the active stresses and membrane potentials within cardiac muscle tissue. However, there exist no methods to reliably measure active stresses, nor do there exist ways to measure transmural membrane potentials that are suitable for in vivo usage. Prior work has devised a linear model to map from the active stresses within the tissue to displacements [1]. In situations where measurements of tissue displacements are entirely precise, we are able to naively solve for the active stresses from the measurements with ease. However, real measurement processes always carry some associated random error and, in the presence of this error, our naive solution to this inverse problem fails. In this work we propose the use of the Ensemble Transform Kalman Filter to more reliably solve this inverse problem. This technique is faster than other related Kalman Filter techniques while still generating high quality estimates which improve on our naive solution. We demonstrate, using in silico simulations, that the Ensemble Transform Kalman Filter produces errors whose standard deviation is an order of magnitude smaller than the least-squares solution.
\end{abstract}

\section{Introduction}

A large number of mathematical models exist that describe the motion and propagation of cardiac electrical potentials and active tissue stresses within the walls of the heart. However, these potentials and stresses are either difficult or impossible to measure, and thus remain difficult to quantify in in vivo settings. Clinical diagnostic tools such as electrocardiograms or electrophysiology studies are generally relied upon to assist in diagnosis of cardiac function. These tools are useful but do not provide the full information on the fundamental cardiac dynamics. Methods like transillumination or optical tomography rely on phototoxic dyes, plunge electrodes provide only local data and are disruptive to the tissue, and noninvasive methods remain unable to produce transmural information, and none of these methods provide information on the active stresses which cause deformation cardiac tissue [2][3].

Following the work of Otani, et al [1], we propose the use of magnetic resonance imaging, ultrasound or computed tomography to non-invasively track the full 3D contractile motion resulting from action potentials (APs) and the mechanical stresses they produce. These techniques all yield high spatial resolution data and, in the case of ultrasound, the high temporal resolution necessary to visualize faster events. Using the linearized Hunter-Nash model [4], we construct an inverse problem whose solution yields the underlying active stress information we desire. However, methods for observing cardiac motion data are inherently uncertain and thus naively solving the inverse problem results in a solution whose uncertainty is magnified.

To combat this uncertainty, we have devised an implementation of the Ensemble Transform Kalman Filter (ETKF) as a state estimation tool [5]. This technique allows us to incorporate knowledge of our flawed models of these real-world systems alongside our uncertain measurements in order to create better estimates of both measured and unmeasured quantities. We utilize the FitzHughNagumo model of AP propagation [6] in conjunction with Nash-Panfilov electromechanical coupling [7] and the Otani, et al model to simulate a 3D region of cardiac tissue in order to study the reconstruction of these APs and active stresses.

Work by Lebert and Christoph [8] utilizes a technique similar to the Kalman Filter, but employ different models for their estimations. Their models and methods encountered difficulties in reconstructing AP and stress data from AP waves moving in directions perpendicular to the direction of the cardiac muscle fibers. Our method is able to faithfully reconstruct full spiral waves with AP motion oriented at any angle relative to the angle of the fiber direction, overcoming this limitation.

\section{Methods}

We first must establish a ground truth, for which we must solve the forward problem. We construct the electrical potential and refractoriness from the FitzHugh- 
Nagumo model, active stress from the Nash-Panfilov model, and then the passive stresses, hydrostatic pressures and strains from the linearized Nash-Hunter model. Random noise is then added to the strains to mimic the inherent noise of the measurement process. The inverse problem is solved using both naive least-squares and the ETKF, with the noisy strains as input data.

\subsection{Cardiac Modeling}

We have chosen to use the FitzHugh-Nagumo model as it is very simple, and thus computationally efficient. The model equations are as follows:

$$
\begin{gathered}
\frac{\partial v}{\partial t}=\Delta v+\frac{1}{\epsilon}\left(v-\frac{v^{3}}{3}-w\right) \\
\frac{\partial w}{\partial t}=\epsilon(v-\gamma w+\beta)
\end{gathered}
$$

$v$ describes the electrical membrane potential at a point in the domain while $w$ describes the refractoriness of the tissue at the same point. $\Delta$ represents the Laplacian, and $\beta=0.7, \gamma=0.8$ and $\epsilon=0.2$ are constant parameters. Using these equations, we are able to construct a base truth AP wave within our domain which adequately simulates the motion of APs in real tissue. We begin by creating a stationary spiral wave pattern in the membrane potential. We then use a version of the Nash-Panfilov coupling model to simulate the production of active stress, $T_{a}$ within the tissue:

$$
\frac{\partial T_{a}}{\partial t}=\epsilon(v)\left(k_{t} v-T_{a}\right) ; \epsilon(v)= \begin{cases}1.1 & \text { for } v<0.1 \\ 1 & \text { otherwise }\end{cases}
$$

This equation allows us to simulate the delay between the $\mathrm{AP}$ and motion in the tissue, as these events do not occur contemporaneously. Finally, we use the linearized HunterNash model to yield tissue displacements from these active stresses. We use the linearized version of the model, as follows:

$$
\begin{gathered}
\frac{\partial}{\partial X_{i}}\left[\frac{1}{2} \frac{\partial T_{i j}}{\partial E_{k l}}(0)\left(\frac{\partial \delta x_{k}}{\partial x}=\frac{\partial \delta x_{Q}}{\partial X_{k}}\right)+T_{a i j}-p \delta_{i j}\right]=0 \\
\frac{\partial \delta x_{j}}{\partial X_{j}}=0
\end{gathered}
$$

In equation $4, X_{i}$ represents the coordinates in the undeformed coordinate system, $T$ is the passive second PiolaKirchoff stress tensor, $E$ is the Lagrange-Green strain tensor, $p$ is the local hydrostatic pressure, $T_{a}$ is the active second Piola-Kirchoff stress tensor due to the force induced by the APs, and $\delta x$ represent the local displacements. The indices $i, j, k, l$ span the values 1,2 , and 3 , representing

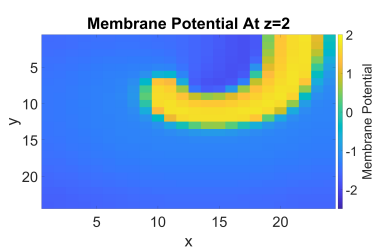

(a)

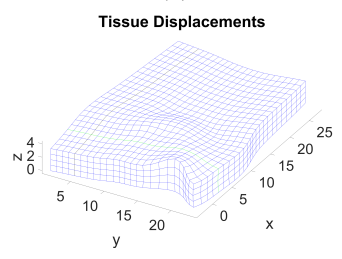

(c)

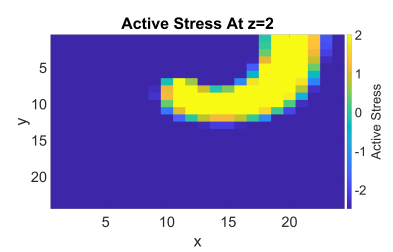

(b)

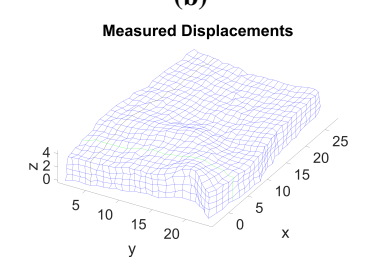

(d)
Figure 1: Figure 1a shows the generated AP from the FitzHugh-Nagumo model in a spiral pattern. From this AP, we use Nash-Panfilov coupling to construct the active stress distribution shown in figure 1b. Then from this stress distribution, we use the Otani et al model to construct the displacements shown in figure 1c. We then add noise to the displacements to simulate measurement error. Note in figure $1 \mathrm{~d}$ that the displacements from $1 \mathrm{c}$ are still clear, though they have been obscured slightly by the noise.

the 3 spatial dimensions. This equation dictates the motion response to an applied active stress profile. Equation 5 enforces incompressibility. In the fully linearized matrix form, we incorporate a further six equations to enforce no displacement of, or rotation about, the center of mass.

For the forward problem, we solve equations (1-5) for $v, w, T_{a}, \delta x, \delta y, \delta z$ and $p$ using forward Euler. A simple finite element method is used to discretize space. The results of this process are shown in figure 1.

With ground truth data defined for all of our variables, we then consider our inverse problem, which solves for $v$, $w, T_{a}$ and $p$ from $\delta x, \delta y$ and $\delta z$. We simulate measurement uncertainty to the displacements by adding a normally distributed random variable with zero mean and covariance corresponding to roughly $10 \%$ of the displacements. Solving the inverse problem naively from these uncertain measurements results in our estimated $T_{a}$ being completely dominated by noise, shown in $2 \mathrm{a}$. It is also of note that this method only allows us to reconstruct $T_{a}$ and $p$, and not the electrical potential or recovery variables. Thus we incorporate the ETKF to improve our results.

\subsection{The Ensemble Transform Kalman Fil- ter}

The Kalman Filter (KF) is a technique which combines information from mathematical dynamical models and uncertain data measured from real-world systems. The ETKF 
is a related technique for nonlinear dynamical systems. The KF, in general, is capable of estimating the entire system based on a measurement of only a subset of the system state.

We first construct an initial ensemble of data estimates. The ensemble can be chosen based on a measurement of the system, but we assume no a priori knowledge of the system state. So each member of the ensemble begins with an initial estimate of being uniformly 0 , plus random noise to ensure that no two ensemble members are exactly the same. We then proceed in two stages: the prediction step and the update step.

For the prediction step, we propagate the ensemble members forward using the dynamics for the system. After some number of prediction steps, we then move to the update step. In the update step, we apply a measurement scheme to the predicted system state in order to generate an expected measurement. We then compare our expected measurement against an actual measurement of the system and construct a gain matrix, which adjusts the entire system state based only on a measurement of part of the entire state, and we use this gain to correct our predicted state.

For our purposes, we have chosen to use 100 ensemble members. We perform 5 prediction steps to every update step, and use update scaling parameter $\rho=1.1$. Our system contains 25 nodes in both the $x$ and $y$ directions, and 5 in the $z$ direction. We set $\Delta x=\Delta y=\Delta z=1$ and $\Delta t=0.01$. We assume that the myocardial fiber direction to run parallel to the $x$-axis. When a segment of tissue is subject to higher active stresses, the tissue contracts in the $x$-direction while expanding in the $y$ - and $z$-directions, as a result of incompressibility.

\section{Results}

We first solve the inverse problem naively. From the noise-afflicted displacement data, we construct a leastsquares solution to attempt to reconstruct the source active stress data, as shown in Figure 2a. We are only able to reconstruct the active stresses, and not any of our other dynamical variables of interest, as we only have a linear relationship between the displacements, active stress and the pressure. The actual wave present in the reconstructed active stress has become heavily obscured due to the presence of noise in the measurements. The error associated with our attempted reconstruction of the active stress is roughly normally distributed and, as of the last time-step, has a mean of $-2.103 \times 10^{-4}$ and a standard deviation of 5.217. This method produces errors that have a small mean, but are highly imprecise and are thus unreliable.

Next, we attempt a solution with the ETKF. We are able to remove much of the noise affecting the displacement data, shown in Figure 2c. We are also able to construct estimates of all of our dynamical variables, not just the active

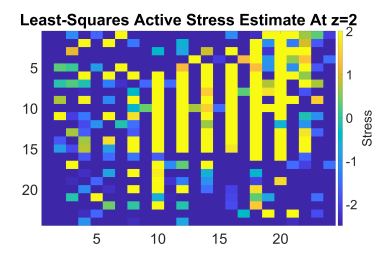

(a)

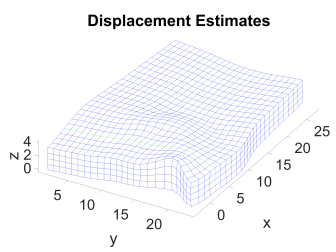

(c)

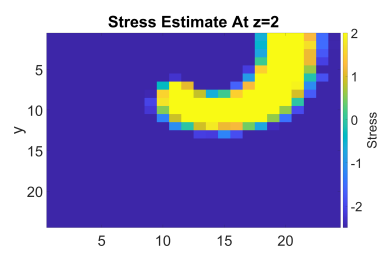

(b)

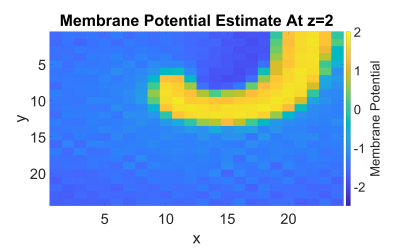

(d)
Figure 2: Figure 2a shows our attempt to solve for the active stress from the noisy displacements using linear leastsquares. The estimated stresses have been lost to noise. Figure $2 b$ shows our estimate of the active stress utilizing the ETKF, which is significantly cleaner than Figure 2a. Compare these two figures to Figure 1b. Also shown are our measurements of the displacements in Figure $2 \mathrm{c}$ and our estimates of the membrane potentials in Figure 2d, obtained from the ETKF. Compare these to Figures 1c and $1 \mathrm{a}$, respectively.

stress. Thus we are additionally able to produce estimates of the membrane potentials and the tissue recovery. Figures $2 \mathrm{a}$ and $2 \mathrm{~b}$ show that our reconstruction of the active stress is much improved compared to that produced by the LSQ solution. The ETKF also produces an estimate of the membrane potential, as shown in $2 \mathrm{~d}$. The error associated with the ETKF active stress estimate remains roughly normally distributed, with mean $5.612 \times 10^{-4}$ and standard deviation 0.282 . Thus, our reconstruction has improved significantly. A side-by-side comparison of the error between the two methods can be seen in Figure 3.

The ETKF is not without limitations. It is significantly slower than the LSQ estimate, and represents a greater need for more high-powered computing. This need could be met by transitioning from the ETKF to the Local Ensemble Transform Kalman Filter, which takes advantage of localization in order to incorporate better parallelization. Additionally, the ETKF has a period of transience after estimations begin. For our simulation, we find that the transient period lasts from $t=0$ until around $t=15$, representing nearly one-third of the total simulation. However, once this transient period ends, the estimations of the system quickly converge towards the true values. Once this convergence occurs, the estimate remains close to the true state. The transient period is less of an issue when reconstructing phenomena on longer time scales. 


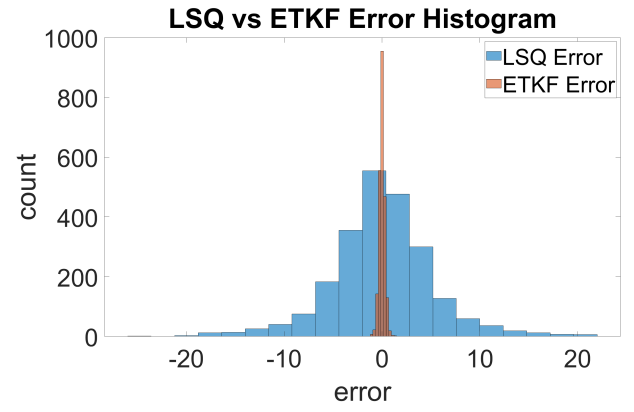

Figure 3: Comparison of the residual error in the estimates of the active stresses between the Least-Squares solution and the ETKF solution. Note that both error distributions appear roughly normal with roughly 0 normal mean, but the ETKF has much smaller variance.

\section{Conclusions}

In this work, we have described further refinements to our novel method for estimating the propagation of APs and active stresses in cardiac fibers and tissues from uncertain measurements of the motion of said tissue. Our method utilizes the ETKF to incorporate uncertain measurements as well as our models to construct estimates of the active stresses, membrane potentials and recovery state within the tissue. We have described an implementation of this model, which represents a three-dimensional segment of tissue, and have shown that it represents significant improvement over the least-squares estimate generated with Matlab's backslash algorithm, both in terms of the number of dynamical variables it can estimate and the accuracy of the estimations that are created.

Our results retain the estimation quality of our prior work while also decreasing computational runtime significantly compared to Beam, et al [9]. Additionally, we have demonstrated a method for reconstructing APs and active stresses even under complex AP configurations, where the direction of motion of the AP runs perpendicular to the direction of the cardiac muscle fibers. In the future, we anticipate using the ETKF or LETKF to create estimates of quantities which may be more difficult to quantify noninvasively, such as tissue fiber direction or various parameters associated with the dynamics of the system. We will also be looking to move to other polyhedral meshes for our domain in order to more accurately represent the whole heart or segments of tissue.

\section{Acknowledgments}

This work has been supported in part by the Computing \& Data-enabled Science and Engineering Award No. 1808530 from the Office of Advanced Cyberinfrastructure of the National Science Foundation.

\section{References}

[1] Otani NF, Luther S, Singh R, Gilmour RF. Transmural Ultrasound-based Visualization of Patterns of Action Potential Wave Propagation in Cardiac Tissue. Annals of Biomedical Engineering October 2010;38(10):3112-3123. ISSN 0090-6964, 1573-9686.

[2] El-Sherif N, Chinushi M, Caref EB, Restivo M. Electrophysiological Mechanism of the Characteristic Electrocardiographic Morphology of Torsade de Pointes Tachyarrhythmias in the Long-QT Syndrome: Detailed Analysis of Ventricular Tridimensional Activation Patterns. Circulation 1997;96(12):4392-4399.

[3] Hillman EMC, Bernus O, Pease E, Bouchard MB, Pertsov A. Depth-resolved Optical Imaging of Transmural Electrical Propagation in Perfused Heart. Optics Express December 2007;15(26):17827-17841. ISSN 1094-4087.

[4] Nash MP, Hunter PJ. Computational Mechanics of the Heart. Journal of elasticity and the physical science of solids July 2000;61(1):113-141. ISSN 1573-2681.

[5] Hunt BR, Kostelich EJ, Szunyogh I. Efficient Data Assimilation for Spatiotemporal Chaos: A Local Ensemble Transform Kalman Filter. Physica D Nonlinear Phenomena June 2007; 230(1):112-126. ISSN 0167-2789.

[6] FitzHugh R. Mathematical Models of Threshold Phenomena in the Nerve Membrane. The bulletin of mathematical biophysics December 1955;17(4):257-278. ISSN 1522-9602.

[7] Nash MP, Panfilov AV. Electromechanical Model of Excitable Tissue to Study Reentrant Cardiac Arrhythmias. Progress in Biophysics and Molecular Biology June 2004; 85(2):501-522. ISSN 0079-6107.

[8] Lebert J, Christoph J. Synchronization-based Reconstruction of Electromechanical Wave Dynamics in Elastic Excitable Media. Chaos An Interdisciplinary Journal of Nonlinear Science September 2019;29(9):093117. ISSN 1054-1500. Publisher: American Institute of Physics.

[9] Beam C, Linte CA, Otani NF. An Implementation of Data Assimilation Techniques for Transmural Visualization of Action Potential Propagation in Cardiac Tissue. In Gimi BS, Krol A (eds.), Medical Imaging 2020: Biomedical Applications in Molecular, Structural, and Functional Imaging. Houston, United States: SPIE. ISBN 978-1-5106-3401-5 978-1-5106-3402-2, March 2020; 57.

Address for correspondence:

Christopher Beam

School of Mathematical Sciences

Rochester Institute of Technology

Rochester, NY 14624

cbb7953@rit.edu 